\title{
Netzwerktheorie(n) - Ein Überblick
}

\author{
Markus Gamper
}

\section{Überblick}

- Netzwerke sind auf der Meso-Ebene anzusiedeln. Sie stehen zwischen dem Individuum (Mikro-Ebene) und den Institutionen (Makro-Ebene).

- Die Theorien gehen von einer Wechselwirkung von Individuum und Netzwerken aus, wobei Beziehungen und ihre gebildeten Strukturen im Fokus der Betrachtung stehen.

- Es gibt nicht „die“ Netzwerktheorie. Vielmehr gibt es verschiedene Theorien oder auch theoretische Ansätze.

- „Grand Theories“ versuchen einen universellen Erklärungsanspruch von Netzwerken auf Akteur*innen zu erklären.

- „Theorien mittlerer Reichweite“ sind forschungsorientierte Arbeitstheorien, die z. B. Netzwerke und ihre Auswirkungen in bestimmte Bereiche wie Gesundheit in den Mittelpunkt stellen.

- „Theorien mittlerer Reichweite“ wie z. B. die strong/weak tie-Theorie finden speziell in der Netzwerkanalyse große Bedeutung und bilden die Grundlage für Hypothesen, oder werden zur Interpretation von Daten verwendet.

M. Gamper $(\bowtie)$

Köln, Deutschland

E-Mail: m.gamper@uni-koeln.de 


\section{$1 \quad$ Netzwerktheorie(n) und ein Einteilungsversuch}

Bezüglich der Netzwerktheorie stellt Scott (2011, S. 24) fest: „[...] theoretical work has long been underdeveloped in social network analysis. While the methods themselves do not require or imply any particular sociological theory, they do require theoretical contextualisation in wider debates". Auch wenn die Theoretisierung von Netzwerken, im Vergleich zu quantitativ-empirischen Verfahren, lange vernachlässigt wurde, gibt es schon seit dem frühen 20. Jahrhundert intensive theoretische Auseinandersetzungen mit dem Konzept der sozialen Beziehungen und ihren Strukturen. Allgemein geht man davon aus, dass Menschen in Beziehungen eingebettet sind und nicht losgelöst von ihren Netzwerken betrachtet werden können. Diese einzelnen Dyaden, das sind Beziehungen zwischen zwei Akteur*innen, verbinden sich wiederum zu größeren Einheiten, sogenannten Netzwerken. Netzwerke sind hierbei auf der Meso-Ebene anzusiedeln. Sie sind damit ein Bindeglied zwischen der Mikro-Ebene, das individuelle Handeln, und der Makro-Ebene, den Institutionen (Weyer 2012, S. 241). Netzwerke bestehen demnach aus Akteur*innen, die Beziehungen untereinander eingehen und damit soziale Strukturen herstellen. Das theoretische Interesse liegt nicht auf den klassischen Attributen von Individuen wie z. B. Geschlecht oder Alter oder auf Merkmale der Institutionen, sondern auf den Beziehungen, deren Strukturen, und der Einbettung der Akteur*innen innerhalb dieser Relationen. Sie bilden den Ausgang der Forschungsfrage, während die genannten Attribute auch mit in die Analyse einbezogen werden.

Hinsichtlich der Netzwerktheorie(n) kann zwischen „Grand Theory“ und „Theorien der mittleren Reichweite“ unterschieden werden. Erstere umfasst ein umfassendes Theoriegebäude mit einem universellen Erklärungsanspruch (Mills 1959), während die „Theorien mittlerer Reichweite“ zwischen Globaltheorien und forschungsorientierten Arbeitstheorien angesiedelt sind. Dies sind Theorien, die auch auf ein spezifisches Forschungsfeld beschränkt bleiben (Merton 1968). Demnach kann man drei Theorieformen in der Netzwerkforschung idealtypisch unterscheiden (Emirbayer 1997; Heidler und Gamper 2017). Als Erstes ist hier der strukturalistische Determinismus zu nennen. Dieser Zweig zieht ausschließlich relationale Merkmale für die Beschreibung von Handlungen der Akteur*innen heran. Demnach ist die Handlung allein durch die Struktur der Netzwerke und die Einbettung des Individuums in diesen Strukturen vordeterminiert. Der strukturalistische Instrumentalismus betont die Handlungsoptionen des Individuums, die sich aus seiner Netzwerkposition ergeben. Diese werden von den Akteure*innen instrumentell, im Sinne eines strukturellen Individualismus, zum eigenen Vorteil (Homo oeconomicus) genutzt. Somit rückt hier stärker als im 
strukturalistischen Determinismus das Individuum in den Blickpunkt des theoretischen Interesses. Der dritte Idealtyp ist der strukturalistische Konstruktivismus. Hier werden soziale Strukturen, kulturelle Determinanten sowie Handlung als eigene Aspekte behandelt. Netzwerke und Individuen sind somit ein Teil der Kultur und beeinflussen diese wiederum. Sie stehen gleichberechtigt nebeneinander. Beziehungen werden als durch die Akteur*innen selbst konstruierte Komponente verstanden, was die Wahrnehmung der beteiligten Akteur*innen nochmals stärker in die Analyse miteinbezieht.

Auch wenn sich die einzelnen Theorien unterscheiden, haben sie doch gemein, dass Relationen und die Einbettung von Individuen das Handeln und die Identität dieser beeinflussen. Auf der anderen Seite zeigen die Theorien aber auch, dass die Akteur*innen Netzwerke knüpfen und diese in Institutionen übergehen und verfestigt werden können. Die Schwerpunkte der Analyse mögen sich zwar unterscheiden, jedoch gehen alle vom Menschen als soziales Wesen aus, welches selbst mit anderen in Netzwerken verbunden ist. Netzwerke können daher wie folgt definiert werden: Netzwerke sind ,[...] as a specific set of linkages among a defined set of persons, with the additional property that the characteristics of these linkages as a whole may be used to interpret the social behaviour of the persons involved" (Mitchell 1969, S. 2).

Im nächsten Abschnitt sollen sowohl „Grand Theories“ und „Theorien mittlere Reichweite" exemplarisch vorgestellt werden, um dieses beschrieben relationale Denken nochmals zu konkretisieren. Der stärkere Fokus liegt jedoch auf den „Theorien mittlerer Reichweite“, da diese in der Empirie eine größere Bedeutung einnehmen.

\section{1 „Grand Theory“ der Netzwerkforschung}

Unter der ersten Gruppe befinden sich Vorläufer der empirischen Netzwerkanalyse (siehe auch Kap. „Netzwerkanalyse - eine methodische Annäherung“). Zu nennen wäre beispielsweise Georg Simmel (1908), der von einer Wechselwirkung von Individuum und Gemeinschaft ausgeht. Simmel spricht hier von sozialen Kreisen: Organische soziale Kreise sind hierbei „Netzwerke“, in die das Individuum hineingeboren wird (z. B. Familie), während rationale Kreise (z. B. Militär oder Wirtschaftsbetriebe), umfasst. Akteur*innen gehen hierbei Beziehungen ein, die wiederum Auswirkungen auf das Handeln der Individuen selber haben und ihnen Möglichkeiten eröffnen oder sie einschränken. Dabei wurden bei Simmel bereits erste einfache Strukturmerkmale herausgearbeitet (Hollstein 2001). 
Ein weiterer wichtiger relationaler Denker ist Sigfried Nadel. Der österreichische Ethnologe unterscheidet zwischen Rolle, Beziehung und sozialer Struktur. Hierbei können soziale Beziehungen einen generellen Charakter (z. B. Freundschaft) besitzen. Sie sind es, die den Wert einer Rolle beschreiben und die Rolle definieren, die wiederum Erwartungen bei den Akteur*innen im Netzwerk hervorruft: „Thus we take ,friendship' to be evidenced by a variety of mutual ways of acting, perhaps visible on different occasions, such as help in economic or emotional re-responses" (Nadel 1957, S. 9).

Für den Soziologen Nobert Elias (2006) spielen Netzwerke für seine Gesellschaftsanalyse eine essenzielle Rolle. Er spricht hier von Figurationen und meint damit, dass Individuen in Dependenzen zueinander stehen und das soziale Miteinander, und damit auch die Akteur*innen selbst, beeinflussen. Ein Netzwerk umfasst ,[...] viele Menschen, die kraft ihrer elementaren Ausgerichtetheit, ihrer Angewiesenheit aufeinander und ihrer Abhängigkeit voneinander auf die verschiedensten Weisen aneinander gebunden sind und demgemäß miteinander Interdependenzgeflechte oder Figurationen mit mehr oder weniger labilen Machtbalancen verschiedener Art bilden" (Elias 2006, S. 15). Dieser offene Mensch („Homines aperti“) besitzt demnach eine relative Autonomie, er ist jedoch von Geburt an andere Akteur*innen ausgerichtet und auf diese in einem Machtgefüge angewiesen (Elias 2006, S. 169).

Eine wichtige prägende Figur in der aktuellen theoretischen Debatte ist wohl der Physiker und Soziologe Harrison White (2008). Mithilfe des relationalen Denkens versucht er zu erklären, wie Akteur*innen ihre Identität entwickeln. Soziale Identitäten, wie beispielsweise Mann/Frau oder Migrant*in/Nicht-Migrant*in, sind nach White nicht gegebene Fakten, sondern werden in sozialen Netzwerken erst konstruiert. Sie sind nicht gegeben, vielmehr werden sie in Bezug zu anderen Akteur*innen in einem bestimmten Netzwerk ausgehandelt. Akteur*innen setzen sich demnach in Relation zu anderen und versuchen, damit ihre Identität zu konstruieren und zu stabilisieren. Diese Strukturen sind dynamisch und bestehen aus sogenannten „stories“, also Geschichten, geteilte Vergangenheiten oder ausgetauschte Erfahrungen, die Akteur*innen übereinander erzählen und miteinander teilen. Diese Geschichten (stories) konstruieren die Identitäten der Akteur*innen- und ihre Beziehungen zueinander und machen uns zu dem, was wir sind (z. B. Mann/Frau).

Die kleine Auswahl an „Grand Theories“ soll die unterschiedlichen relationalen Denkweisen kurz vorstellen und spiegelt die Debatte in sehr verkürzter Form wider: Netzwerke werden als Grundlage gesellschaftlichen Handelns oder auch von Identität gesehen. Sie sind der Ursprung des Handelns oder werden durch Handlung beeinflusst und besitzen einen universellen Erklärungsanspruch. 


\subsection{Netzwerktheorien der mittleren Reichweite}

Neben den bereits erwähnten „Grand Theories“ existieren auch kleinere „relationtheoretische Ansätze“ mittlerer Reichweite. Deren Fokus liegt zwar auch auf den sozialen Beziehungen und ihren Strukturen, diese Erklärungsmodelle sind jedoch enger gefasst. Zum Teil können die hier folgenden Ansätze auch unter diese gröBeren Theorien subsumiert werden. Es sind theoretische Konzepte, die explizit aus der Netzwerkforschung stammen und auf dieses spezifische Forschungsfeld beschränkt sind. Diese können, bevor eine Studie durchgeführt wird, helfen, Forschungsfragen zu konzipieren, oder auch Ergebnisse zu interpretieren. Einige möchten wir im folgenden Kapitel vorstellen. Dabei haben wir uns für Konzepte entschieden, die in vielen Studien auch empirisch umgesetzt werden. Hierbei handelt es sich nicht um eine vollständige Liste von Konzepten, vielmehr bietet die Auflistung einen Einblick in die konzeptionellen Herangehensweisen. Darunter fallen die theoretischen Ansätze, wie beispielsweise von starken und schwachen Beziehungen, strukturellen Löchern, Homophilie, Beliebtheit, Reziprozität, Balance-Theorie und Cliquen. Viele dieser Ideen werden im Kap. „Netzwerkanalyse - eine methodische Annäherung“" wieder aufgegriffen.

\subsubsection{Strong/weak ties}

Ein sehr wichtiges relationales Konzept geht auf Granovetter (1973) zurück. In seinem Ansatz unterscheidet der Soziologe zwei Arten von Beziehungen anhand ihrer Intensität: starke (strong) und schwache (weak) Beziehungen (ties). Die Differenzierung zwischen starken und schwachen Beziehungen ist demnach ,(probably linear) combination of the amount of time, the emotional intensity, the intimacy (mutual confiding) and the reciprocal services [...]" (Granovetter 1973, S. 1361). Starke Relationen sind geprägt durch beispielsweise Reziprozität, hohe Kontaktintensität, hohe Intimität und Emotion sowie Vertrauen. Darunter fallen z. B. Familienmitglieder oder auch gute Freund*innen. „Weak ties“ hingegen sind lose Beziehungen mit z. B. geringer Kontaktfrequenz, geringer Intimität und Multiplexität. Zu nennen wären hier beispielsweise Urlaubsbekanntschaften, Kolleg*innen und lose Bekanntschaften. Während starke Beziehungen für Aspekte, wie z. B. emotionale Unterstützung von Bedeutung sind, konnte Granovetter zeigen, dass schwache Beziehungen ihren ganz eigenen Nutzen besitzen. Sie ermöglichen einen Zugang zu neuen Informationen und Ressourcen, die nicht aus dem sozialen Nahbereich kommen, da diese, anders als starke Beziehungen, Brücken in andere Subgraphen schlagen (siehe Abb. 1). Dies ist damit begründet, dass diese Beziehungen Pfaddistanzen reduzieren und man dadurch mit Personen in 


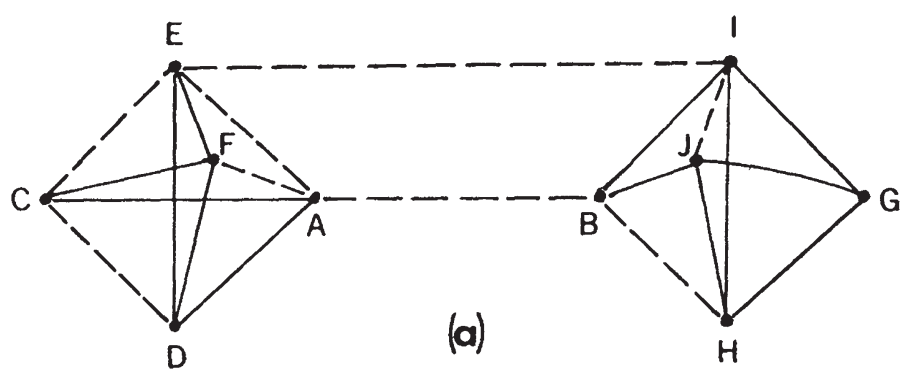

Abb. 1 Die Bedeutung von schwachen Beziehungen nach Granovetter. (Quelle: Granovetter 1973, S. 1365)

Kontakt kommt, die andere Informationen als das enge soziale Umfeld besitzen, welche uns beispielsweise hinsichtlich Interessen und Wissen sehr ähnlich sind. In seiner Studie „Getting a Job“ (Granovetter 1985) konnte Granovetter nachweisen, dass bei der Suche nach einer Arbeitsstelle im Ingenieurbereich besonders schwache Beziehungen Erfolg versprechen. Diese sind sogar von gröBerer Bedeutung als klassische Bewerbungsverfahren. Dieses theoretische Konzept ist in der Gesundheitsforschung von Interesse, wenn davon ausgegangen wird, dass unterschiedliche Beziehungsarten unterschiedliche Konsequenzen auf das Gesundheitsverhalten oder auch das subjektive Wohlbefinden nach sich ziehen. Bei einer solchen Einschätzung muss empirisch (qualitativ oder auch quantitativ) das Konzept und die Unterscheidung immer von Anfang an mitgedacht und definiert werden. $\mathrm{Zu}$ nennen wären hier Studien über die Auswirkung von schwachen Beziehungen und die Diffusion von Selbstmordgedanken (Baller und Richardson 2009) oder die vermittelnde Rolle von starken oder schwachen Beziehungen zwischen Armut, Gesundheit und Wohlbefinden (Cattell 2001). Eine genaue Unterscheidung zwischen beiden Beziehungsarten ist oft nicht eindeutig und kann sich auch von Kontext zu Kontext unterscheiden. Daher ist es für die eigene Forschung wichtig, Parameter für die Unterscheidung deutlich herauszuarbeiten und zu begründen.

\subsubsection{Strukturelle Löcher}

Wie Granovetter beschäftigt sich auch Burt (1992) mit Beziehungsarten und deren unterschiedlichen Wirkungen. Während Granovetter eher den Fokus auf die Intensität der Relation fokussiert, ist für Burt $(1992,2004)$ die Struktur und damit die Position eines Akteurs bzw. einer Akteurin im Netzwerk von essenzieller 


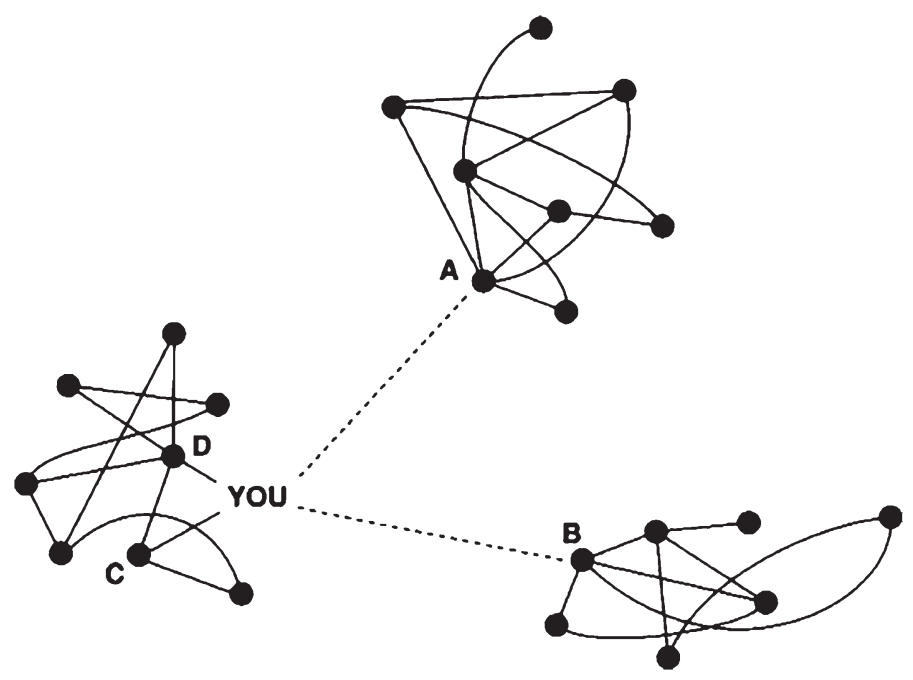

Abb. 2 Strukturelle Löcher. (Quelle: Burt 1992, S. 27)

Bedeutung: ,[...] people have an advantage because of their location in a social structure" (Burt 2004, S. 351). Damit thematisiert er die strukturelle Einbettung der Akteur*innen und den daraus erwachsenden Handlungsmöglichkeiten sowie -restriktionen. Mit strukturellen Löchern sind hierbei fehlende Beziehungen gemeint, die zwei oder mehr Subgraphen trennt (siehe Abb. 2). Diese Löcher verhindern beispielsweise die Weitergabe von Informationen oder anderen Ressourcen. Akteur*innen, die solche Löcher schließen, indem sie Subgraphen verbinden und eine Brückenfunktion einnehmen, können durch ihre strukturelle Verortung Vorteile besitzen. Ein Vorteil ist beispielsweise der Einblick in sehr unterschiedliche Subgraphen, der es erlaubt, nicht redundante Informationen zu generieren bzw. Wissen aus unterschiedlichen Bereichen zusammenzuführen. In seiner sogenannten „Broker“-Position kann er den Fluss von Informationen nach seiner Sicht gestalten (z. B. tertius gaudens ${ }^{1}$ ). Er kann beispielweise regulieren, welche Informationen wann und wie in welches Subnetzwerk weitergegeben werden und welche nicht (Scheidegger 2010).

${ }^{1}$ Damit ist der lachende Dritte gemeint. 
In der Gesundheitsforschung findet man diese Theorie nur selten wieder. $\mathrm{Zu}$ nennen wäre z. B. die egozentrierte Studie von Cornwell (2009). Der Autor geht der Frage nach, inwieweit sich der Gesundheitszustand auf die Überbrückung solcher strukturellen Löcher auswirkt. Dabei stellt der Autor fest, dass kognitive und funktionelle Gesundheit positiv signifikant mit der Überbrückung solche Löcher verbunden sind. Die Studie von Schafer (2013) geht hier einer ähnlichen Frage nach und unterstützt die Ergebnisse von Cornwell (2009).

\subsubsection{Homophilie (Einfluss/Selektion)}

Ein wichtiges Konzept in der Netzwerkforschung ist das der Homophilie. Der Begriff, wie wir ihn heute kennen, stammt von Lazarsfeld und Merton (1954), die Beobachtungen klassischer Netzwerkstudien und Forschungen der Ethnologie zur Ehebildung miteinander verbanden. Vereinfacht bedeutet dies: „Gleich und gleich gesellt sich gern“. Hierbei unterscheiden die beiden Autoren zwischen „status homophily“ und „value homophily“. Erstere bezieht sich sowohl auf zugeschriebene Merkmale wie beispielsweise Ethnie, Geschlecht als auch auf Religion und Bildung. „Value homophily“ bezieht auch auf Personen, die ähnliche Einstellungen oder Denkweisen besitzen, losgelöst von Status der jeweiligen Person. Hinsichtlich der Netzwerke bedeutet dies, dass sich Menschen Beziehungen zu Personen aufbauen, die einen ähnlich sind. McPherson et al. (2001) stellen dabei fest, dass besonders Ethnie, Alter, Religionszugehörigkeit, Bildung, Geschlecht und Beruf Faktoren sind, die innerhalb von Netzwerken wichtig sind: „Homophily in race and ethnicity creates the strongest divides in our personal environments, with age, religion, education, occupation, and gender following in roughly that order" (McPherson et al. 2001, S. 415). Wie es zur Herstellung von Homophilie in Netzwerken kommt, kann anhand zweier unterschiedlicher Prozesse erklärt werden. Zum einen geschieht dies durch Selektionsprozesse. Hier suchen sich die Akteur*innen, die sich aufgrund von einem oder mehreren Attributen ähnlich sind, gegenseitig aus und bilden ein Netzwerk. Zum anderen können sich Akteur*innen suchen, die sich in einem oder mehreren Merkmalen unterscheiden, sich aber im Austausch immer mehr angleichen (Knecht 2008). Auch wenn das Ergebnis ein hoher Homophilie-Wert ist, sind die Prozesse doch different. In der Gesundheitsforschung geht man beispielsweise der Frage nach, inwieweit sich Jugendliche in ihrem Rauchverhalten beeinflussen oder sich selektiv zusammentun (Knecht 2008). Um diese beiden genannten Effekte empirisch unterscheiden zu können, sind zwei Messzeitpunkte notwendig. 


\subsubsection{Beliebtheit: Popularität und preferential attachement}

Eine weitere Theorie der mittleren Reichweite ist die der „Beliebtheit“. In diesem Fall „besitzen“ manche Akteur*innen mehr Beziehungen als andere und sind damit beliebter. Hierbei möchten wir zwei Ansätze unterscheiden. Zum einen das Konzept, das davon ausgeht, dass bestimmte Attribute (z. B. Alter, Geschlecht, Gesundheit) sich auf die Beliebtheit von Akteur*innen auswirken, und zum anderen kann es sein, dass jemand, der bereits viele Beziehungen „besitzt“, aufgrund dieser noch mehr Beziehungsanfragen bekommt. Ersteres kann hier als Popularität bezeichnet werden. Populäre Akteur*innen sind demnach diejenigen, mit denen viele Akteur*innen eine Beziehung eingehen wollen bzw. eingehen. Erste Forschungen konnten zeigen, dass intelligente, extrovertierte und leistungsfähige Schüler*innen populärer sind als andere (Bonney 1946; Young und Cooper 1944). Vor dem Hintergrund des Gesundheitsverhaltens stellen Valente et al. (2005) heraus, dass besonders beliebte Studierende anfangen zu rauchen. „Popular middle school students were more likely to become smokers compared to their less popular peers" (Valente et al. 2005, S. 323).

Beim preferential attachement geht man auch davon aus, dass Beziehungen innerhalb eines Netzwerkes ungleich verteilt sind. Die Prämisse beruht hier jedoch auf den Beziehungen, d. h. das Akteur*innen, die bereits schon viele Beziehungen vereinigen, noch leichter Beziehungen hinzubekommen. Merton (1968) beschreibt dies als „Matthäus-Prinzip“ („Wer hat, dem wird gegeben“). Dieses Phänomen konnte z. B. De Solla Price (1976) anhand von Zitationen in Artikeln (,cumulative advantage“) und Barabási und Albert (1999) für soziale und sozial-technologische Netzwerke (Filmschauspieler*innen, Stromnetz, Internet) (,scale-free“) herausstellen.

\subsubsection{Reziprozität}

Ein wichtiges Grundprinzip, auf dessen Basis es zu unterschiedlichen Formen sozialer Netzwerke kommen kann, ist die Reziprozität (Prinzip der Gegenseitigkeit). Dieses besagt, dass Menschen für eine bestimmte erbrachte Leistung auch eine entsprechende Gegenleistung erwarten. Hier kommt es zum Prinzip von Gabe und Gegengabe (Mauss 2000) und die daraus entstehenden Netzwerke. Diese Erwartung macht viele Formen der sozialen Interaktion überhaupt erst möglich. Simmel geht so weit zu behaupten, dass es ein Grundprinzip von Gesellschaften ist (Simmel 1908 [1989]; s. oben). Hierbei sind Transaktionen zwischen den Akteur*innen nicht immer an einen Preis oder an formale Regeln gebunden. Eine Gegenleistung muss auch nicht in selber Form wie die erhaltene Leistung 
beglichen werden, dennoch sollte sie als mindestens adäquat und ähnlich von dem/der Gabenempfänger*in empfunden werden. Leistet man beispielsweise Freunden Hilfe bei einem Umzug, so kann erwartet werden, später eine ähnliche Leistung zu erhalten. Werden die Erwartungen beim Gabengebenden nicht erfüllt, wird die Reziprozitätsnorm verletzt und dauerhafter sozialer Austausch weniger wahrscheinlich. Reziprozitätsnormen sind von Kultur, historischen Aspekten und der Rolle der involvierten Akteur*innen (z. B. Freund*in, Bekannte*r) abhängig. Zum Beispiel ist man innerhalb der Familie in sogenannten westlichen Staaten für enge Freunde oder Verwandte eher bereit, eine Leistung zu erbringen, ohne eine zeitlich (un-)mittelbare entsprechende Gegenleistung zu erwarten (Sahlins 1999). Ein Recht (z. B. Vertragsrecht) existiert in der Regel nicht. Die Struktur des Austausches zwischen den jeweiligen Tauschpartner*innen kann durch quantitative Netzwerkforschung sehr gut abgebildet werden, Verpflichtungsnormen und Praktiken stehen eher der qualitativen Forschung offen (s. Kap. „Netzwerkanalyse - eine methodische Annäherung"). Beispielweise geht die qualitative Studie von Wentowski (1981) „Reciprocity and the Coping Strategies of Older People: Cultural Dimensions of Network Building“ den Fragen nach 1) wie kulturelle Regeln den Austausch von Unterstützung innerhalb der Netze regeln, und 2) wie Unterschiede in der Art und Weise, wie ältere Menschen diese Regeln bei der Schaffung von Unterstützung im Laufe der Zeit erfahren, interpretieren. Die Forschung zeigt ferner die große persönliche Bedeutung der Gegenseitigkeit für die Erhaltung des psychischen Selbstwertgefühls älterer Menschen. Dennoch stellen Abbott und Freeth (2008) heraus, dass das theoretische Modell der Reziprozität in der Gesundheitsforschung kaum Berücksichtigung findet.

\subsubsection{Balance-Theorie}

Eine psychologische Theorie, die in der Netzwerkforschung eine große Bedeutung einnimmt, ist die der Balance in Triaden. Die Balance-Theorie geht vor allem auf die Gleichgewichtstheorie, die der Konsistenztheorie ${ }^{2}$ zugerechnet $^{2}$ werden kann, zurück. Demnach versuchen Akteur*innen Einstellungen und Überzeugungen widerspruchsfrei zu gestalten (Witte 1989). Vor diesem Hintergrund befasst sich die Gleichgewichtstheorie mit Widersprüchen von Beziehungsstrukturen zwischen Akteur*innen untereinander oder zwischen Akteur*innen und

${ }^{2}$ Die Konsistenztheorie geht davon aus, dass Harmonien in kognitiven Prozessen - wie z. B. Wahrnehmung, Einstellungen - eine positive Wirkung auf das Individuum haben. 

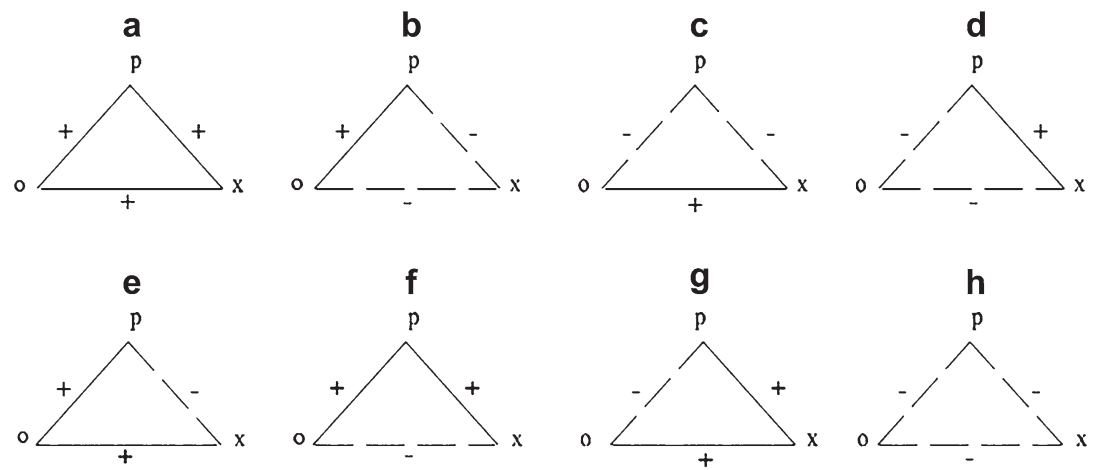

Abb. 3 Unbalancierte und balancierte Triaden. (Quelle: Durchgezogene, mit „, “ markierte Linien sind positive Beziehungen, gestrichelte, mit ,-- " markierte Linien sind negative Relationen. Die Triaden (a)-(d) sind demnach balanciert, die Triaden (e)-(g) sind unbalanciert. Triade (h) ist „unbestimmt“. (Manhart 1995, S. 120))

anderen Elementen (Manhart 1995). Es wird davon ausgegangen, dass Akteur*innen nach einem Gleichgewicht streben. Mit dem Gleichgewichtszustand ist ,[...] eine Situation gemeint, in der die Relationen zwischen den Größen harmonisch zueinander passen; es gibt keinen Drang zu einer Veränderung " (Heider 1977, S. 238). Aus der von Heider (1958) entwickelten Balancetheorie lassen sich Aussagen zu Effekten über Beziehungen ableiten. Der Psychologe ging von folgender Konstellation aus:

1. Eine Person P, eine Person O und einer Situation, ein Ereignis, eine Idee oder eine Sache (X).

2. Es gibt positive und negative Beziehungen.

3. Individuen streben danach, einen Zustand der Balance zu erreichen.

Wann ist nun eine Triade ausbalanciert? Sie ist dann ausbalanciert, wenn alle Beziehungen positiv oder zwei Beziehungen negativ und eine positiv sind. Dies soll anhand eines Beispiels erklärt werden. Nehmen wir an, Person A ist mit Person B befreundet. Gleichzeitig ist Person B aber mit Person C in einer negativen Beziehung. Eine Balance wäre z. B. dann gegeben, wenn Person A auch mit Person $\mathrm{C}$ in einem Konflikt stehen würde. In anderen Worten: Der Feind meines Freundes ist mein Feind. Ein Ungleichgewicht liegt dann vor, wenn eine Beziehung negativ und zwei Relationen positiv sind. Die unterschiedlichen Konstellationen werden in Abb. 3 nochmals dargeboten. 
Die Studie von Cornwell (2009) stellt heraus, dass schlechte Gesundheit Auswirkung auf das Ego-Alter-Alter-Beziehungssystem haben kann. Welche Beziehungen in Triaden überbrückt werden können und welche nicht, muss jedoch noch weiterhin erforscht werden.

\subsubsection{Kleingruppen - Beispiel die Clique}

Kleingruppenforschung nimmt in der Netzwerkforschung eine etablierte und wichtige Stellung ein. Unter einer Kleingruppe versteht man eine überschaubare Anzahl von Akteur*innen, die jeweils miteinander in Kontakt treten können (Homans 1951). Diese Verbindungen sind von besonderem Interesse, da sich dort soziale Mechanismen leichter erforschen lassen. Hierzu gehört z. B. die Clique. „Im alltäglichen Sprachgebrauch bezeichnet der Begriff Clique gemeinhin eine Gruppe von Individuen, welche in relativ engen, direkten und wechselseitigen Beziehungen stehen. Der gemeinsame Zweck, den die Gruppenmitglieder einer Clique verfolgen, kann dabei sehr verschieden sein: Eine Straßengang, ein Kaffeekränzchen oder ein Stammtisch sind nur einige Beispiele für derartige Kleingruppen " (Täuber 2010, S. 397).

Hierbei sind Cliquen ein Teil eines größeren Netzwerkes. Sie sind damit dichte Verbindungen zwischen Akteur*innen in einem Netzwerk. Diese Teilgruppen, so die Annahme, zeigen neben der engen Verbundenheit auch andere Merkmale, wie z. B. hohe Aktivität innerhalb des kleineren Verbundes, große Solidarität und Identifikation untereinander. Damit bilden Cliquen oder auch andere Kleingruppen innerhalb eines Netzwerkes einen eng verbundenen Subgraph bzw. Teilgraph; also ein Netzwerk in einem größeren Netzwerk. Vor dem Hintergrund der Netzwerkforschung kann z. B. eine Clique wie folgt definiert werden: „Eine Clique ist ein maximaler (knotengenerierter) vollständiger Teilgraph. Innerhalb einer Clique sind also alle Knoten durch einen Pfad der Länge 1, d. h. direkt, verbunden " (Trappmann et al. 2005, S. 74). Berechnungsverfahren werden wir im Methodenkapitel noch kennenlernen. Bezüglich der Einbindung in eine Clique und der Gesundheit zeigt die Studie von Ennett und Bauman (1993), dass Schüler*innen, die in Cliquen eingebunden sind, nicht so häufig rauchen wie isolierte Akteur*innen. Auf die psychische Gesundheit stellen Provan und Sebastian (1998) heraus, dass eine Eingebundenheit in ganze Netzwerke eher einen negativen Effekt hat, während die Einbindung in Cliquen, die sich auch überlappen, eher positiv wirken können.

Mit diesem Teil sollte verdeutlich werden, dass neben den „Großen-Theorien“ auch empirisch-orientierte Ansätze existieren. Hierbei ist es wichtig, sich vor der empirischen Umsetzung des eigenen Projekts zu überlegen, welche der 
relationalen Aspekte für die Erklärung oder das Verstehen des zu untersuchenden Phänomens in der eigenen Studie von Bedeutung sind. Hierfür gibt es bereits bestimmte, getestete Möglichkeiten unter denen ausgewählt werden kann (siehe Kap. „Netzwerkanalyse - eine methodische Annäherung“). Wichtig ist hier jedoch, dass diese immer im Bezug zur Gesundheit oder auch zum Gesundheitsverhalten in Beziehung gesetzt werden sollten.

\section{Soziale Netzwerke und Theorie - Ein Fazit}

Netzwerktheorien stellen die Einbettung von Individuen in ihre soziale Umwelt in den Fokus der Betrachtung. Sie gehen davon aus, dass das Handeln bzw. Identitäten und Institutionen in Wechselwirkung mit sozialen Strukturen stehen. Netzwerke werden somit der Mesobene zugerechnet, die zwischen dem individuellen Handeln und den Institutionen verortet sind (Weyer 2012). Wie dieses Kapitel auch gezeigt hat, gibt es jedoch nicht „die eine“ Netzwerktheorie. Allgemein kann in drei unterschiedliche Ausrichtungen unterschieden werden: der strukturalistische Determinismus, der strukturalistische Instrumentalismus und der strukturalistische Konstruktivismus (Emirbayer und Goodwin 1994). Hierbei unterscheiden die Autoren vor allem zwischen dem Bedeutungsanspruch von Relationen für das Verständnis sozialer Phänomene. Ferner variieren sie auch hinsichtlich ihres Erklärungsanspruches. Während die „Grand Theories“ einen allumfassenden Ansatz beanspruchen, mit dem ein universeller Erklärungsanspruch verfolgt wird, bleiben die ,Theorien der mittleren Reichweite“ auch auf ein spezifisches Forschungsfeld beschränkt. Auch wenn sogenannte „Grand Theories“ existieren und auch neuere Ansätze wissenschaftlich große Bedeutung haben, finden diese in der empirischen Forschung nur begrenzt Beachtung. Hier kommen vor allem Theorien mittlerer Reichweite zur Anwendung (siehe Kap. „Netzwerkanalyse - eine methodische Annäherung“). Zu nennen sind hier z. B. die strong/ weak tie-Theorie, die Theorie der strukturellen Löcher, die Triaden-Theorie, Balance-Theorie, die Theorie der Homophilie, Cliquen-Theorie oder auch die der Reziprozität. Die Theorien helfen, die Entstehung von Netzwerken, dessen Wirkung oder ihre Bedeutung für die Akteur*innen zu erklären oder diese besser zu verstehen. Sie dienen auch als Grundlage für die Hypothesenbildung. Welche der Theorien für die eigene Forschung relevant sein könnte, kann hier nicht geklärt werden; die Aufzählung soll jedoch helfen, einen Überblick zu gewinnen, um dann selbst Theorien für das eigene Forschungsvorgehen auszuwählen. 


\section{Leseempfehlungen}

McPherson, M., Smith-Lovin, L., \& Cook, J. M. (2001). Birds of a feather: Homophily in social networks. Annual review of sociology, 27 (1), S. 415444. Ein englischer Artikel zum Thema Homophilie, der einen guten Überblick zum Thema gibt.

White, H. C. (2008). Identity and control: How social formations emerge - second edition (H. C. White, Hrsg.). Princeton: Princeton University Press. Eine der wohl aktuellsten und spannendsten Theoriearbeiten zum Thema Netzwerke.

Emirbayer, M. (1997). Manifesto for a relational sociology. American journal of sociology, 103 (2), S. 281-317. Englischer Artikel, der der Frage nach der Bedeutung von relationalem Denken und relationalen Ideen in der Soziologie auf den Grund geht.

Burt, R. S. (2004). Structural holes and good ideas. American Journal of Sociology, 110, S. 349-399. Ein Artikel, der mit Daten aus der Organisationssoziologie die Idee der strukturellen Löcher und ihre Bedeutung mit einem empirischen Beispiel sehr gut beschreibt.

Granovetter, M. S. (1973). The strength of weak ties. The American Journal of Sociology, 78, S. 1360-1380. Der wohl bekannteste Artikel zum Thema schwache Relationen und deren Wert.

\section{Literatur}

Abbott, S., \& Freeth, D. (2008). Social capital and health: Starting to make sense of the role of generalized trust and reciprocity. Journal of Health Psychology, 13(7), 874-883.

Baller, R. D., \& Richardson, K. K. (2009). The "dark side" of the strength of weak ties: The diffusion of suicidal thoughts. Journal of Health and Social Behavior, 50(3), 261-276.

Barabási, A.-L., \& Albert, R. (1999). Emergence of scaling in random networks. Science, 286, 509-512.

Bonney, M. E. (1946). A sociometric study of the relationship of some factors to mutual friendships on the elementary, secondary, and college levels. Sociometry, 9, 21-47.

Burt, R. S. (1992). Structural holes: The social structure of competition. Cambridge: Harvard University Press.

Burt, R. S. (2004). Structural holes and good ideas. American Journal of Sociology, 110(2), 349-399.

Cattell, V. (2001). Poor people, poor places, and poor health: The mediating role of social networks and social capital. Social Science and Medicine, 52(10), 1501-1516. 
Clyde Mitchell, J. (1969). Social networks in urban situations: Analyses of personal relationships in central african towns. Manchester: Manchester University Press.

Cornwell, B. (2009). Good health and the bridging of structural holes. Social Networks, 31(1), 92-103.

De Solla Price, D. J. (1976). A general theory of bibliometric and other cumulative advantage processes. Journal of the American Society for Information Science, 27(5), 292-306.

Elias, N. (2006). Was ist Soziologie. Weinheim: Juventa. (Erstveröffentlichung 1970).

Emirbayer, M. (1997). Manifesto for a relational sociology. American Journal of Sociology, 103(2), 281-317.

Emirbayer, M., \& Goodwin, J. (1994). Network analysis, culture, and the problem of agency. American Journal of Sociology, 99(6), 1411-1454.

Ennett, S. T., \& Bauman, K. E. (1993). Peer group structure and adolescent cigarette smoking: A social network analysis. Journal of Health and Social Behavior, 34(3), 226-236.

Granovetter, M. S. (1973). The strength of weak ties. The American Journal of Sociology, 78, 1360-1380.

Granovetter, M. S. (1985). Getting a job: A study of contacts and careers. Chicago: University of Chicago Press.

Heider, F. (1958). The psychology of interpersonal relations. New York: Wiley.

Heider, F. (1977). Psychologie der interpersonalen Beziehungen. Stuttgart: Klett.

Heidler, R., \& Gamper, M. (2017). Ein Freund, ein guter Freund? Oder: Über blinde Flecken der stochastischen Modellierungsverfahren ERGM und SIENA am Beispiel von Freundschaften in Schulklassen. In H. Löwenstein \& M. Emirbayer (Hrsg.), Netzwerke, Kultur und Agency. Problemlösungen in relationaler Methodologie und Sozialtheorie (S. 355-380). Weinheim: Beltz Juventa.

Hollstein, B. (2001). Grenzen sozialer Integration. Zur Konzeption informeller Beziehungen und Netzwerke. Opladen: Leske \& Budrich.

Homans, G. C. (1951). The human group. Transaction: Piscataway.

Knecht, A. B. (2008). Friendship selection and friends' influence. Dynamics of networks and actor attributes in early adolescence. Utrecht University. https://dspace.library. uu.nl/handle/1874/25950

Lazarsfeld, P. F., \& Merton, R. K. (1954). Friendship as a social process: A substantive and methodological analysis. In M. Berger, T. Abel, \& C. Page (Hrsg.), Freedom and Control in Modern Society (S. 18-66). New York: Van Nostrand.

Manhart, K. (1995). KI-Modelle in den Sozialwissenschaften: Logische Struktur und wissensbasierte Systeme von Balancetheorien. München: Oldenbourg.

Mauss, M. (2000). The gift: The form and reason for exchange in archaic societies (Reissue Aufl.). New York: Norton.

McPherson, M., Smith-Lovin, L., \& Cook, J. M. (2001). Birds of a feather: Homophily in social networks. Annual Review of Sociology, 27(1), 415-444.

Merton, R. K. (1968). The Matthew effect in science. Science, 159, 56-63.

Mills, C. W. (1959). The sociological imagination. Oxford: Oxford University Press.

Nadel, S. F. (1957). The theory of social structure. London: Routledge.

Provan, K. G., \& Sebastian, J. G. (1998). Networks within networks: Service link overlap, organizational cliques, and network effectiveness. Academy of Management Journal, 41(4), 453-463. 
Sahlins, M. D. (1999). Zur Soziologie des primitiven Tauschs. Berliner Journal für Soziologie, 9(2), 149-178.

Schafer, M. H. (2013). Structural advantages of good health in old age: Investigating the health-begets-position hypothesis with a full social network. Research on Aging, 35(3), 348-370.

Scheidegger, N. (2010). Strukturelle Löcher. In C. Stegbauer \& R. Häußling (Hrsg.), Handbuch Netzwerkforschung (S. 145-155). Wiesbaden: VS.

Scott, J. (2011). Social network analysis: Developements, advances, and prospects. Social Network Analysis and Mining, 1, 21-26.

Simmel, G. (1908). Soziologie. Untersuchungen über die Formen der Vergesellschaftung. Berlin: Duncker \& Humblot. (Erstveröffentlichung 1989).

Täube, V. G. (2010). Cliquen und andere Teilgruppen sozialer Netzwerke. In C. Stegbauer \& R. Häußling (Hrsg.), Handbuch Netzwerkforschung (S. 397-406). Wiesbaden: VS.

Trappmann, M., Hummell, H. J., \& Sodeur, W. (2005). Strukturanalyse sozialer Netzwerke, Konzepte, Modelle, Methoden. Wiesbaden: VS.

Valente, T. W., Unger, J. B., \& Johnson, C. A. (2005). Do popular students smoke? The association between popularity and smoking among middle school students. Journal of Adolescent Health, 37(4), 323-329.

Wentowski, G. J. (1981). Reciprocity and the coping strategies of older people: Cultural dimensions of network building. The Gerontologist, 21(6), 600-609.

Weyer, J. (2012). Soziale Netzwerke: Konzepte und Methoden der sozialwissenschaftlichen Netzwerkforschung. München: Oldenbourg.

White, H. C. (2008). Identity and control: How social formations emerge (2. Aufl.). Princeton: Princeton University Press.

Witten, E. (1989). Quantum field theory and the Jones polynomial. Communications in Mathematical Physics, 121(3), 351-399.

Young, L. L., \& Cooper, D. H. (1944). Some factors associated with popularity. Journal of Educational Psychology, 35, 513-535.

Open Access Dieses Kapitel wird unter der Creative Commons Namensnennung 4.0 International Lizenz (http://creativecommons.org/licenses/by/4.0/deed.de) veröffentlicht, welche die Nutzung, Vervielfältigung, Bearbeitung, Verbreitung und Wiedergabe in jeglichem Medium und Format erlaubt, sofern Sie den/die ursprünglichen Autor(en) und die Quelle ordnungsgemäß nennen, einen Link zur Creative Commons Lizenz beifügen und angeben, ob Änderungen vorgenommen wurden.

Die in diesem Kapitel enthaltenen Bilder und sonstiges Drittmaterial unterliegen ebenfalls der genannten Creative Commons Lizenz, sofern sich aus der Abbildungslegende nichts anderes ergibt. Sofern das betreffende Material nicht unter der genannten Creative Commons Lizenz steht und die betreffende Handlung nicht nach gesetzlichen Vorschriften erlaubt ist, ist für die oben aufgeführten Weiterverwendungen des Materials die Einwilligung des jeweiligen Rechteinhabers einzuholen.

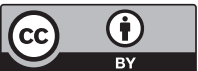

